\title{
OUTCOME AFTER AZACITIDINE TREATMENT IN PATIENTS WITH HIGH-RISK MYEOLODYSPLASTIC SYNDROME, CHRONIC MYELOMONOCYTIC LEUKEMIA TYPE 2 AND ACUTE MYELOID LEUKEMIA - A SINGLE CENTER EXPERIENCE (PRELIMINARY DATA)
}

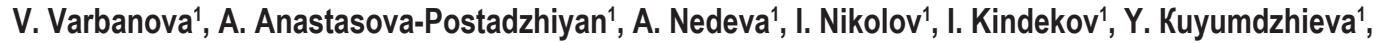
N. Petkova', E. Vikentieva², M. Lubomir ${ }^{3}$, J. Raynov ${ }^{1}$

${ }^{1}$ Clinic of Hematology, Military Medical Academy - Sofia, Bulgaria

'Laboratory of clinical immunology, , Military Medical Academy - Sofia, Bulgaria

${ }^{3}$ Laboratory of Cytogenetics and Molecular Biology, Military Medical Academy - Sofia, Bulgaria

\begin{abstract}
Introduction: Hypomethylating agents have become a standard therapy for certain myeloid malignancies. Aim: The aim of this preliminary study was to assess efficacy and safety of azacitidine in patients with myelodysplastic syndromes (MDS), chronic myelomonocytic leukemia with 10-29\% blasts (CMML-2) and acute myeloid leukemia (AML) treated in a single center. Material and Methods: Twenty-six (69\% male and 31\% female, median age 67.8 years) patients ( $M D S,=15$; CMML-2, $n=2$; $A M L, n=9$ ) treated with azacytidine in the period April 2017 to October 2018 year were included in the study. Disease assessment was performed after the 3rd cycle, 6th cycle, and at progression. Results: The median number of administered cycles was 6 (1-16). Erythroid response was achieved in $46.7 \%$ after 3 rd cycle and $66.7 \%$ after 6 th cycle. Platelet response was reached in $72.7 \%$ after $3 \mathrm{rd}$ cycle and $40 \%$ after 6 th cycle and neutrophil hematological improvement in $27.3 \%$ and $50 \%$, respectively. Only one patient (8.3\%) progressed after the 6th cycle, stable disease or better marrow response was achieved in the others. The median progression free survival (PFS) and overall survival (OS) were 7.9 and 10.7 months in the MDS group and 9.7 and 11.5 months in the AML group, respectively. None of the patients with CMML-2 progressed at the end of the study. The only found factor to correlate with shortened PFS and OS was IPSS high risk MDS. The most frequent grade $\geq 3$ adverse events was neutropenia $38.5 \%$, followed by anemia $15.4 \%$ and thrombocytopenia $11.5 \%$. Conclusion: The therapy with azacitidine is an option for elderly patients with high-risk MDS, AML and CMML-2 that provides PFS and OS for approximately one year irrespective of age or nosological subgroup. These are preliminary data and larger patient cohort and longer follow-up period are needed for clinical conclusions.
\end{abstract}

Key words: azacytidine, MDS, AML, CMML-2

Corresponding author: Viktoria Plamenova Varbanova, Clinic of Hematology, Military Medical Academy, Multiprofile Hospital for Active Treatment, 3 Sv. Georgi Sofiyski Blvd., 1606 Sofia, Bulgaria, tel.: +359 884 674160, e-mail: viktoriia1982@abv.bg 


\section{INTRODUCTION}

$\mathrm{M}$ yelodysplastic syndromes (MDS) include myeloid neoplasia characterized by the presence of dysplastic changes in one or more myeloid lines, peripheral cytopenia, and a tendency for progression to acute myeloid leukemia (AML) [1]. MDS is a heterogeneous disease with different clinical manifestations and time to AML transformation which determines the need for different therapeutic behavior and purpose in each individual case in accordance with generally accepted risk stratification systems [2, 3].

The therapeutic efficacy of different molecules with proven efficacy for treatment of acute myeloid leukemia were investigated in order to improved survival of patients with MDS. However, some patients die from causes related to the accompanying cytopenia or due to progression to AML, or from other non-hematologic events [4].

December 2008, the Europepean Medicines Agency (EMA) approved the hypomethylating agent azacitidine for the treatment of certain myeloid neoplasia [5], based on the results of randomized trials [6,7]. The therapeutic efficacy of azacitine has been associated with reduced aberrant hypermethylation and a direct cytotoxic effect [8].

\section{AIM}

The aim of this preliminary study was to assess the efficacy and safety of azacitidine in patients with
MDS, CMML-2 and AML by analyzing the therapeutic response, progression-free survival (PFS) and overall survival (OS) for each group of treated patients.

\section{MATERIAL AND METHODS}

Clinical records of 26 patients, treated with azacitidine from April 2017 to October 2018 in the Clinic of Hematology at Military Medical Academy, Sofia were retrospectively reviewed. The mean age of the patients was $67.8(58-80)$ years. The distribution by gender was as follows: 18 (69\%) males and $8(31 \%)$ females. Indications for azacitidine therapy were MDS with intermediate-2 and high-risk determined by the International Prognostic Scoring System (IPSS) [2], chronic myelomonocytic leukemia-2 (CMML-2) and AML patients ineligible for intensive chemotherapy and allogeneic haematopoietic stem cell transplantation. The characteristics of the patients in the subgroups are presented in Table 1.

The distribution according to cytogenetic risk determined by IPSS and the revised IPSS-R [2, 3] are presented in Figure 1.

Azacitidine was applied subcutaneously at a dose of $75 \mathrm{mg} / \mathrm{m}^{2}$ for 7 consequent days. Blood counts were done before azacytidine administration, then monthly. Bone marrow evaluation was done by cytological or histological assessment of blast infiltration. Disease assessment was performed on the 3rd and the 6th month after initiation of the treatment, or at progression. Response was evaluated according to

Table 1. Patients characteristics $(n=26)$

\begin{tabular}{|c|c|c|c|c|c|}
\hline Показател & $\begin{array}{c}\text { All patients } \\
n(\%)\end{array}$ & \multicolumn{2}{|r|}{$\begin{array}{l}\text { MDS } \\
\mathrm{n}(\%)\end{array}$} & $\begin{array}{l}\text { AML } \\
\mathrm{n}(\%)\end{array}$ & $\begin{array}{c}\text { CMML-2 } \\
n(\%)\end{array}$ \\
\hline $\mathrm{n}$ & 26 & \multicolumn{2}{|c|}{$15(57.7 \%)$} & $9(34.6 \%)$ & $2(7.7 \%)$ \\
\hline sex & $\begin{array}{c}\mathrm{m}: 18(69 \%) \\
\mathrm{f}: 8(31 \%)\end{array}$ & \multicolumn{2}{|c|}{$\begin{array}{c}\text { m: } 9(60 \%) \\
f: 6(40 \%)\end{array}$} & $\begin{array}{l}\text { m: } 7(78 \%) \\
f: 2(22 \%)\end{array}$ & m: $2(100 \%)$ \\
\hline aged & $\begin{array}{c}67.8 \\
(58-80) \\
\end{array}$ & \multicolumn{2}{|c|}{$\begin{array}{c}66.5 \\
(58-80)\end{array}$} & $\begin{array}{c}67.4 \\
(59-73) \\
\end{array}$ & $\begin{array}{c}79.5 \\
(79-80) \\
\end{array}$ \\
\hline $\begin{array}{l}\text { Prior therapy before } \\
\text { azacitidine }\end{array}$ & & \multicolumn{2}{|c|}{$2(13.3 \%)^{*}$} & $5(55.6 \%)^{\star *}$ & $1(50 \%) \#$ \\
\hline & & \multicolumn{2}{|c|}{\begin{tabular}{c|c} 
WHO & IPSS \\
$\operatorname{RCMD}(n=4)$ & intermediate- $(n=4)$ high \\
& $(n=0)$ \\
RAEB I $(n=2)$ & intermediate-2 $(n=2)$ \\
& high $(n=0)$ \\
RAEB II $(n=9)$ & intermediate-2 $(n=7)$ \\
high $(n=0)$
\end{tabular}} & $\begin{array}{c}\text { FAB } \\
\text { AML-M2 }(n=3) \\
\text { AML-M4 }(n=3) \\
\text { AML }-M 5(n=3)\end{array}$ & \\
\hline
\end{tabular}

Abbr.: RAEB: refractory anemia with excess of blasts; RCMD: refractory cytopenia with multilineage dysplasia*: one patient with low dose Ara-C and one patient with 6-mercaptopurine; ${ }^{* *}$ : conventional induction therapy; \# hydroxycarbamide; m: male; f: female 


\section{IPSS-cytogenetic risk}

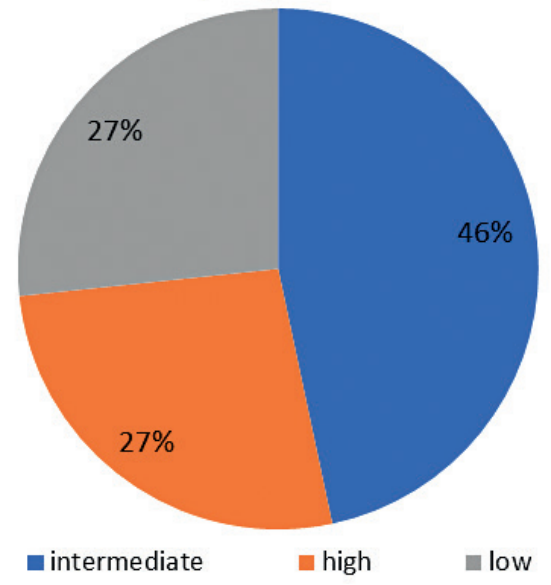

IPSS-R cytogenetic risk

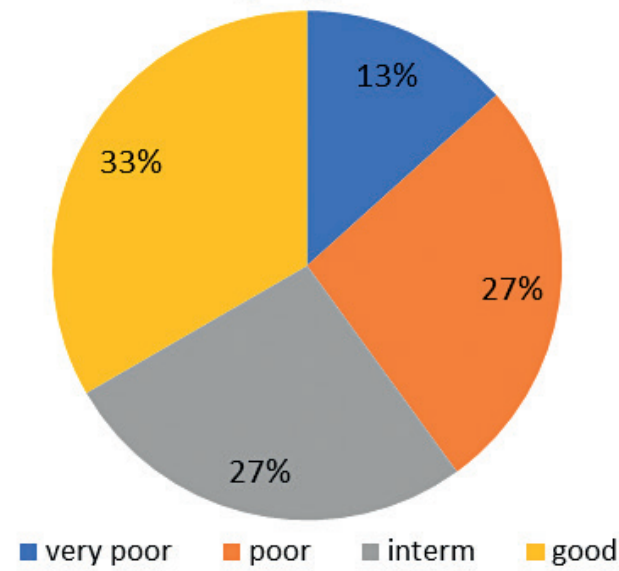

Fig. 1. Cytogenetic profiles in patients with MDS according to IPSS and IPPS-R. Abbr.: IPSS: high risk $(n=4)$ : complex karyotype $(n=3)$ and del $(7)(n=1)$; low risk $(n=4)$ : normal karyotype $(n=3)$ and del $(5 q)(n=1)$, intermediate risk $(n=7)$ due to missing data $(n=1)$ or aberrations not fit for low or high risk cytogenetics $(n=6): 46 X Y$, der $(20) t(1 ; 20)(q 21 ; q 13)(n=1),+8(n=1)$, del $(20 q)(n=1),+21(n=2),+C(n$ $=1)$. IPSS-R: good risk $(n=5)$ : normal karyotype $(n=3)$, del $(5 q)(n=1)$, del $(20 q)(n=1)$; intermediate risk $(n=4):+8(n=1),+21(n=2)$, $+C(n=1)$; high risk $(n=4)$ : $\operatorname{del}(7)(n=1)$, two aberrations or missing data $(n=3)$ and very high risk $(n=2)$ based on complex karyotype

the International Working Group (IWG) response criteria in myelodysplasia [9, 10]. Categorical variables were analyzed by the $\chi^{2}$ or the Fisher's exact tests. OS and PFS was defined as the survival from the first day of azacitidine administration until death from any cause or progression, respectively or last followup. OS and PFS data were analyzed by the log-rank test and Kaplan-Meier estimates were computed. All statistical analysis was performed using SPSS, v. 19.

\section{RESULTS}

Between April 2017 and October 2018 in the Clinic of Hematology at Military Medical Academy, Sofia, 26 patients were treated with azacitidine. According to the WHO classification 9 patients were with refractory anemia with excess of blasts II (RAEB II), 1 with RAEB I, 4 with refractory cytopenia with multilineage dysplasia (RCMD), 2 with CMML-2 and 9 patients with AML. Median age was 67.8 years (range 58-80) as $62 \%$ of the patients were over 65 years old. According to IPSS patients with MDS are stratified as $13.3 \%$ high risk $(n=2)$ and $86.7 \%$ intermediate $-2(n=13)$ (Tabl. 1).

High risk $A M L$ is determined in 5 patients due to refractory disease after intensive induction or FLT3-ITD mutation. Four AML patients are assigned as intermediate risk because of luck of information from cytogenetic analysis.

Azacitidine is applied as first line therapy in $65.4 \%$ of the patients $(n=17)$ and as a second line in $34.6 \%(n=9)$ (Tabl. 1). Azacitidine was given for a median of six cycles (1-
16): respectively MDS: 6 (1-11), AML: 6 (1-16) and CMML-2: 11 (8-14).

\section{Azacitidine therapeutic efficacy}

Disease assessment was performed on the 3rd and the 6th month after inition of the treatment or at progression or death. Thirteen patients completed 6 cycles of azacitidine ( 7 patients with MDS, 2 with CMML-2 and 4 with AML), 7 patients did not complete the whole 6 cycles due to progression (4 MDS, 3 OML), 4 (3 MDS, 1 AML) because of death and 2 patients (1 MDS, $1 \mathrm{AML}$ ) did not complete the 6 cycles due to a delay.

\section{Marrow complete response}

Marrow complete response after 3 cycles was assessed according to the information from 23 patients, 3 patients with MDS were excluded due to lack of bone marrow status data (Fig. 2).

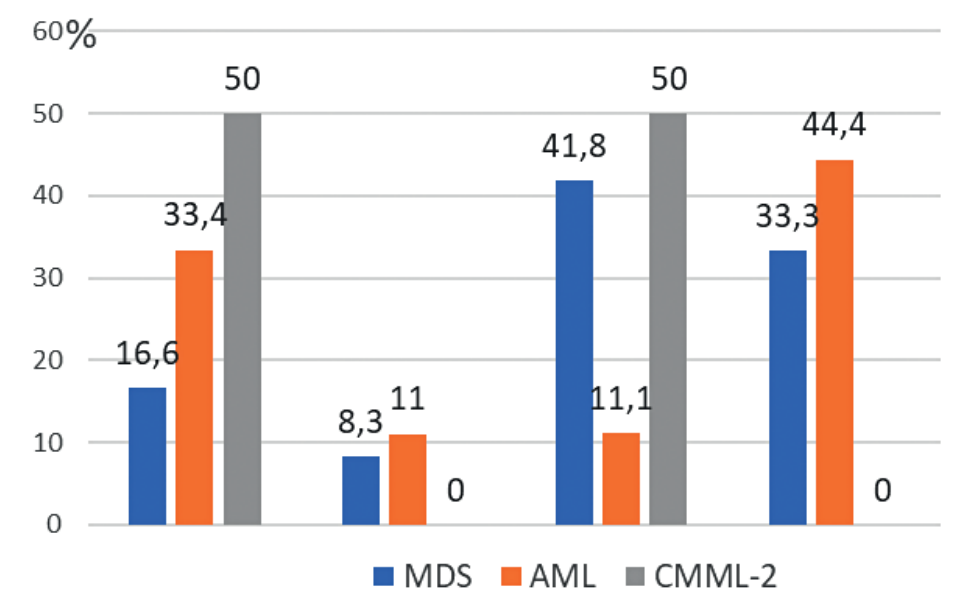

Fig. 2. Marrow response after 3 cycles azacytidine 
Marrow response after 6 cycles of azacytidine treatment was assessed in 12 patients (the other patients progressed/died or were still in the middle of the treatment regimen or bone-marrow status data were missing). Progression disease was reported only in one patient with AML (8.3\%), in the other $91.7 \%$ was achieved stable disease or better response.

\section{Hematologic improvement}

Hematologic response was evaluated in 18 patients. Three patients with MDS and 5 with AML were excluded because of inability to accomplish the 3rd cycle or due to missing data. Six cycles of azacytidine treatment have been completed in 14 patients (MDS, $\mathrm{n}=8$; CMML-2, $\mathrm{n}=2, \mathrm{AML}, \mathrm{n}=4$ ).

\section{Erythroid response}

Hematological improvement $(\mathrm{HI})$ of hemoglobin after 3rd cycle was achieved in $33.3 \%$ of the patients from MDS group, $100 \%$ from CMML-2 group and $75 \%$ in AML (Fig 3). After 6th cycle $\mathrm{HI}$ of hemoglobin was achieved in $66.7 \%$ of MDS group, $100 \%$ of CMML-2 group and $66.7 \%$ of AML (Fig 3 ). All patients with $\mathrm{HI}$ became transfusion-independent.

\section{Platelet response}

$\mathrm{HI}$ of platelets was reported in $66.7 \%$ of MDS patients, $50 \%$ of CMML-2 and $75.0 \%$ of AML after 3 rd cycle and $42.8 \%$ of MDS, $100 \%$ of CMML-2 and $75.0 \%$ of AML after 6th cycle (Fig. 4)

\section{Neutrophil response}

Patients with CMML-2 were excluded from the analysis due to neutrophil counts prior to azacitidine therapy above $1 \times 10^{9} / \mathrm{l}$. After cycle $3, \mathrm{HI}$ of neutrophils was reported in $14.3 \%(n=2)$ in the MDS group and $25 \%(n=1)$ in AML, corresponding to $27.3 \%$ of all patients analyzed. After the 6th cycle, $\mathrm{HI}$ of neutrophils was achieved at $14.3 \%(n=2)$ and $25 \%(n=1)$ of MDS and AML, respectively, or a total of $50.0 \%$ for the entire population treated.

\section{Survival}

At the end of the follow-up 11 (42.3\%) patients were alive, of which 6 were continuing azacytidine therapy. The other $15(57.7 \%)$ died due to disease progression. None of the patients with CMML-2 progressed or died within the study period.

The median PFS and OS were 7.9 months $(95 \% \mathrm{Cl}$ 5.5-10.4) and 10.7 months $(95 \% \mathrm{Cl} 8.2-14.7)$ for MDS/CMML-2 group and 7.9 months $(95 \% \mathrm{Cl} 5.6$ 13.8) and 11.5 months $(95 \% \mathrm{Cl} 6.5-15.6)$ for AML, respectively (fig. 5).

No influence on PFS or OS was observed depending on group type (MDS/CMML-2 vs AML) or aged (< 65 vs. $\geq 65$ years old). Negative impact on PFS and OS was found according to IPSS risk stratification in MDS patients' group: high risk IPSS vs. intermediate-2, 1.05 months vs 7.9 months, $p=0.01$ (PFS) and 1.4 months vs. 12.39 months, $p=0.01$ (OS).

\section{Azacitidine safety assessment}

All patients were included in the safety analysis. Anemia grade 3 was reported in $15.4 \%$ (MDS, $n=1$ and AML, $n=1)$. Thrombocytopenia of any grade were reported in $11.5 \%$ (MDS, $n=1 ; C M M L-2, n=1$ and AML, $n=1)$. Gr. 3 thrombocytopenia was seen in one patient and gr. 4 in 2 patients (AML, $n=1 ; C M M L, n$ =1). The most frequent hematology toxicity was neutropenia $-42.3 \%$ (MDS, $n=9$; AML, $n=2$ ), gr. 3 in 3 patients with MDS and gr. 4 in 5 patients with MDS and 2 with AML.

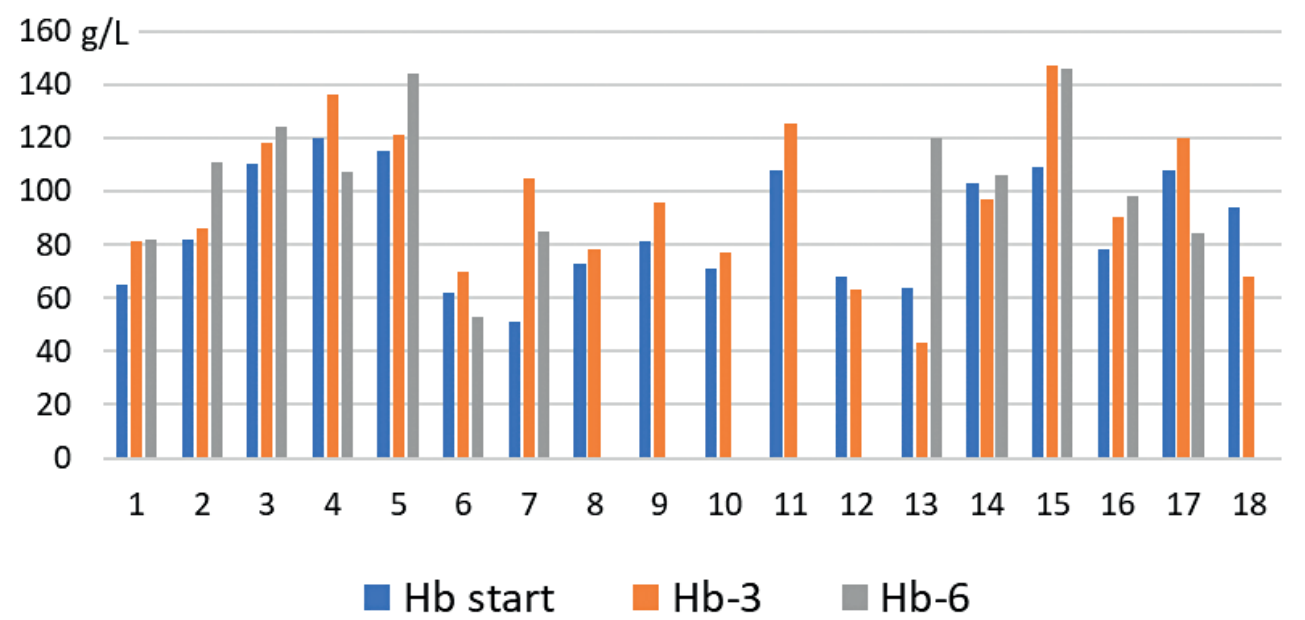

Fig. 3. Levels of hemoglobin $(\mathrm{Hb}, \mathrm{g} / \mathrm{l})$ measured prior to treatment $(\mathrm{Hb}$-start) and on the 3rd $(\mathrm{Hb}-3)$ and the 6 th $(\mathrm{Hb}-3)$ month 


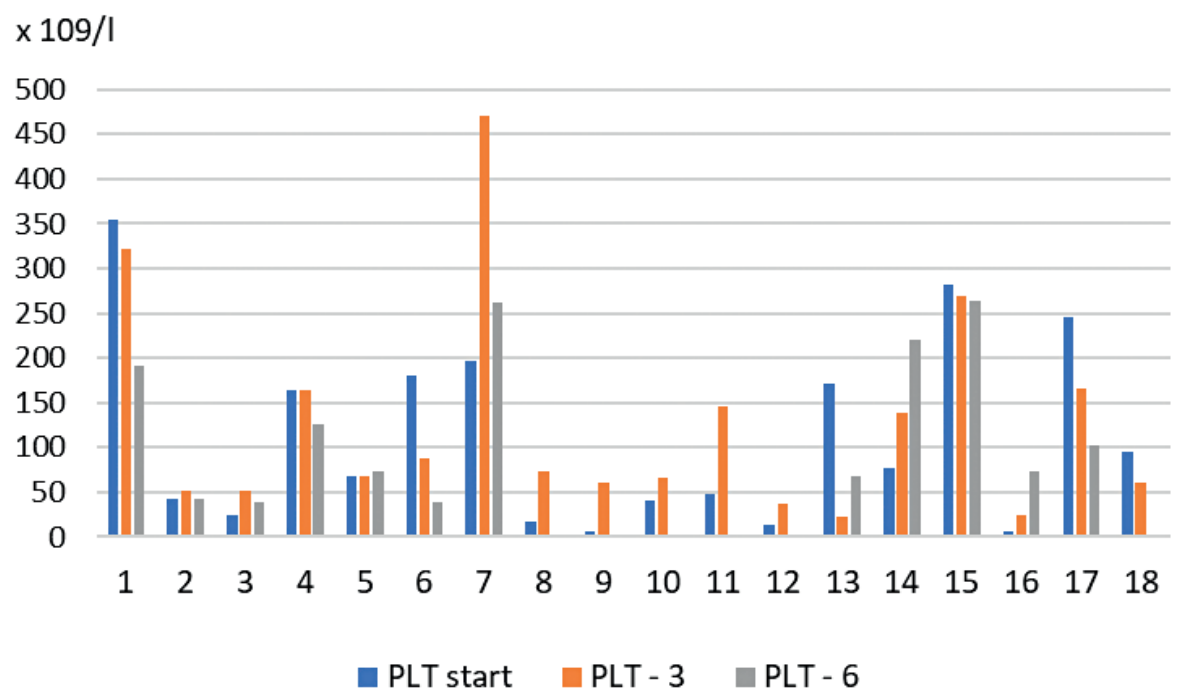

Fig. 4. Levels of thrombocytes (x109/I ) measured prior to treatment (PLT-start), and on the 3rd (PLT-3) and the 6th (PLT-6) month.
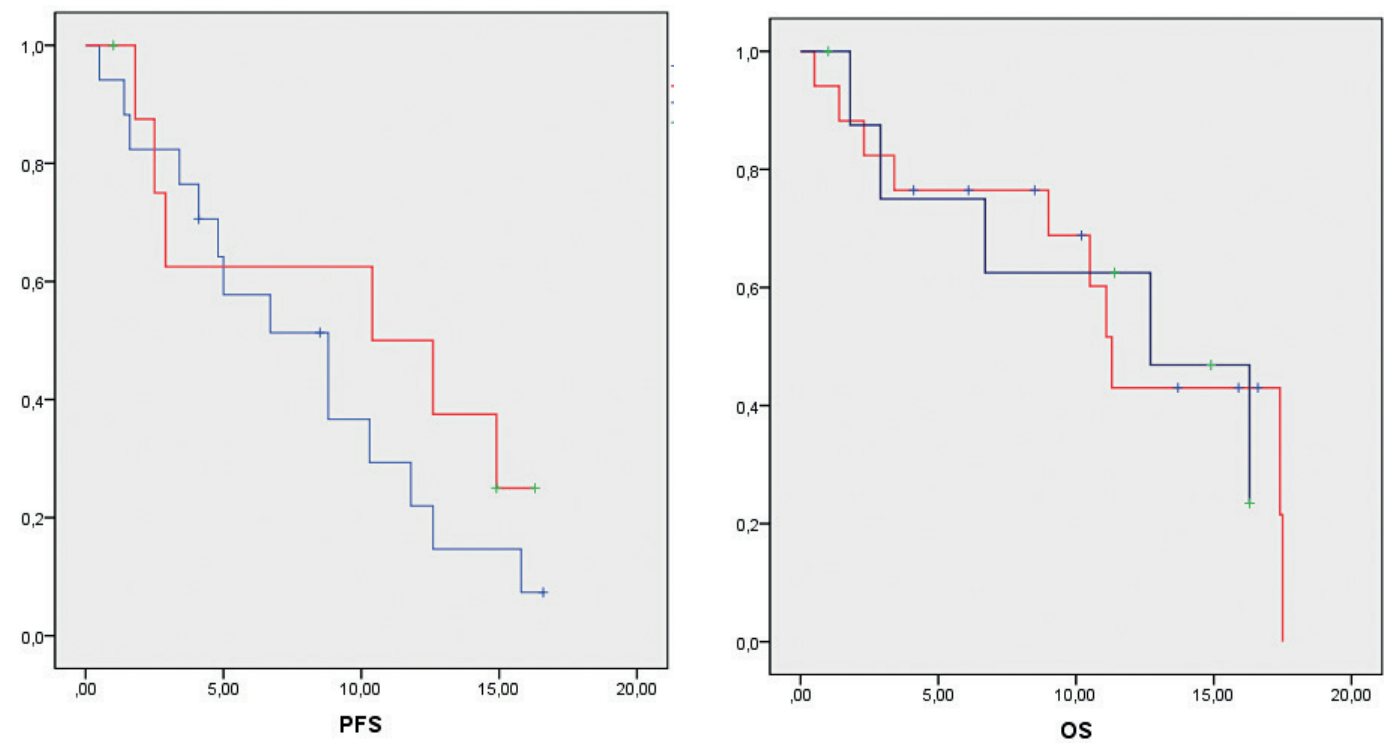

Fig. 5. Progression free survival and overall survival, months (- MDS/CMML-2; - AML)

\section{DISCUSSION}

Myelodysplastic syndromes comprise a heterogeneous group of malignant hematopoietic stem cell disorders that differ by clinical presentation and prognosis. Patients with high risk MDS (IPSS intermediate-2 and high) manifest with faster transformation in AML and shorter OS compared to low risk MDS (IPSS low and intermediate-1) [2]. Until the approval of azacitidine for clinical practice, therapeutic strategies in high-risk patients provided a transformation-free survival less than one year and OS approximately 15.0 months [6, 7]. Silvermanet et al [6] first demonstrated improvement of PFS with azacytidine compared to best supported care (BSC) in MDS pa- tients. Subsequently Fenaux P. et al [7] confirmed the therapeutic efficacy of azacitidine in high risk MDS and CMML-2 compared to BSC (9.6 months benefit for OS and 4.9 months for leukemia free survival, LFS), low dose Ara-C (9.2 months benefit for OS and 0.5 months for LFS) and convention intensive therapy for AML (OS extension by 9.4 months and 2.4 months for LFS). Also, azacitidine significantly improved OS in patients $\geq 65$ years old, compared to standard chemotherapy (3.9 months benefit) [11]

In the presented study the median OS was 10.8 months. No significant differences in PFS and OS were found depending on the subgroup: MDS/ CMML-2 vs AML. The median OS for AML was 11.5 months which is similar to that reported by Dombret 
$\mathrm{H}$ et. al [11] (10.4 months) but higher than the reported by Micheva et al. [12] (5.4 months). However, it should be noted that unlike the other two studies in our group, only $66.7 \%$ of patients with AML were $\geq 65$ years of age, although age did not show statistical significance for OS and azacitidine was first-line therapy only in $33.3 \%$ of our patients which does not allow comparison of results.

The found median OS from 10.7 months in MDS/ CMML-2 group is similar to those reported from Micheva et al. for intermediate-2/high risk MDS - 12.6 months [12]. The observed significant negative effect of high risk MDS for OS and PFS confirms the importance of risk stratification for MDS and the current need for more effective therapeutic approaches. However, transfusion independence was achieved in $46.7 \%$ of patients after 3 cycles of azacitidine and in $66.7 \%$ after 6 cycles. Platelet $\mathrm{HI}$ was observed in at least $50.0 \%$ of patients treated with azacitidine after 3 months and 6 months of therapy. Hematological improvement of neutrophils at $27.3 \%$ and $50.0 \%$, respectively, after the 3rd and 6th cycle. At the same time, azacitidine therapy has been associated with the occurrence of adverse events, as neutropenia was reported the most frequent similarly to other researches [6].

\section{CONCLUSION}

The therapy with azacitidine is a standard therapeutic approach in patients with MDS IPSS intermediate-2 and high-risk, CMML-2 and AML ilegible for intensive chemotherapy. In our study, it was confirmed that azacitidine provided PFS and OS for approximately one year, regardless of age or nosological subgroup. Comparison of results and clinical findings requires the accumulation of a larger patient cohort and a longer follow-up period.

Disclosure summary: The authors have nothing to disclose.

\section{REFERENCES}

1. WHO classification of Tumours of Haematopoetic and Lymphoid Tissues 4th Edition:88

2. Greenberg P, Cox C, LeBeau MM, et al. International scoring system for evaluating prognosis in myelodysplastic syndromes. Blood, 1997; 89(6):2079-2088.

3. Schanz J, Tüchler H, Solé F, et al. New Comprehensive Cytogenetic Scoring System for Primary Myelodysplastic Syndromes (MDS) and Oligoblastic Acute Myeloid Leukemia After MDS Derived From an International Database Merge. J Clin Oncol, 2012: 30(8): 820-829.

4. Dayyani F, Conley AP, Strom SS, et al. Cause of death in patients with lower-risk myelodysplastic syndrome. Cancer, 2010; 116(9):2174-2179.

5. https://www.ema.europa.eu/en/documents/product-information/vidaza-epar-product-information_en.pdf

6. Silverman LR, Demakos EP, Peterson BL, et al. Ranomized controlled trial of azacitidine in patients with the myelodysplastic syndrome: a study of the cancer andl eukemia group B. J ClinOncol. 2002; 20(10):2429-40.

7. Fenaux P, Mufti GJ, Hellström-Lindberg E, et al. Efficacy of azacitidine compared with that of conventional care regimens in the treatment of higher-risk myelodysplastic syndromes: a randomised, open-label, phase III study. Lancet Oncol. 2009;10(3):223-32.

8. Santini V, Melnick A, Maciejewski JP, et al. Epigenetics in focus: pathogenesis of myelodysplastic syndromes and the role of hypomethylating agents. CritRevOncol Hematol. 2013;88(2):231-45.

9. Cheson BD, Greenberg P, Bennett JM, et al. Clinical application and proposal for modification of the International Working Group (IWG) response criteria in myelodysplasia. Blood,. 2006;108(2):419-425.

10. Genova M, Grudeva-Popova J, Gercheva-Kiuchukova L, et al. Guide for the diagnosis and treatment of hematological diseases. Bulgarian Medical Society of hematology, Art Tracer, Varna 2018: ISBN 978-619-7094-39-8: 215-232.

11. Dombret $\mathrm{H}$, Seymour JF, Butrym A, et al. International phase 3 study of azacitidine vs conventional care regimens in older patients with newly diagnosed $\mathrm{AML}$ with $>30 \%$ blasts. Blood. 2015;126(3):291-9.

12. Micheva I, Gerov V, Dimitrova S, et al. Outcome after azacitidine treatmentinpatients with high-risk myelodysplastic syndrome and acute myeloid leukemia in the Clinic of Hematology at St. Marina University Hospital, Varna. Scripta Scientifica Medica, 2017; 50 (1): 31-35.

Revised: 15 December 2019, Accepted: 20 December 2019 